\title{
MULTIPLICATION OF STRONGLY SUMMABLE SERIES
}

\author{
by A. V. BOYD
}

(Received 30th July, 1958) relations

1. Introduction. Given a series $\sum_{n=0}^{\infty} a_{n}$ we define $A_{n}, A_{n}^{(k)}, E_{n}^{(k)}(k>-1)$, by the

$$
A_{n}^{(k)}=\sum_{\nu=1}^{n}\left(\begin{array}{c}
k+n-\nu \\
n-\nu
\end{array}\right) a_{v}, \quad E_{n}^{(k)}=\left(\begin{array}{c}
k+n \\
n
\end{array}\right), \quad A_{n}=A_{n}^{(0)} .
$$

The series $\Sigma a_{n}$ is said to be summable $(C, k)$, where $k>-1$, to the sum $s$ if

$$
a_{n}^{(k)}=A_{n}^{(k)} / E_{n}^{(k)} \rightarrow s \quad \text { as } n \rightarrow \infty ;
$$

to be summable $(C,-1)$ to $s$ if it converges to $s$ and $n a_{n}=o(1)$; to be absolutely summable $(C, k)$, or summable $|C, k|$, to $s$ if it is summable $(C, k)$ to $s$ and

$$
\sum_{n=1}^{\infty}\left|a_{n}^{(k)}-a_{n-1}^{(k)}\right|<\infty
$$

and to be strongly Cesàro summable to $s$ with order $k>0$ and index $p$, or summable $[C ; k, p]$ to $s$, if

$$
\sum_{n=0}^{N}\left|a_{n}^{(k-1)}-s\right|^{p}=o(N)
$$

Hyslop [1] has shown that necessary and sufficient conditions for $\Sigma a_{n}$ to be summable $[C ; k, p]$, where $k>0, p \geqslant 1$, to the sum $s$ are that it be summable $(C, k)$ to the sum $s$ and that

$$
\sum_{n=1}^{N} n^{p}\left|a_{n}^{(k)}-a_{n-1}^{(k)}\right|^{p}=o(N)
$$

These conditions suggest that summability $[C ; 0, p]$ be defined as convergence together with the condition

$$
\sum_{n=0}^{N} n^{p}\left|a_{n}\right|^{p}=o(N)
$$

and on the basis of this definition Hyslop [1] has proved the inclusion theorem that summability $[C ; k, p] \quad(k \geqslant 0, p \geqslant 1)$ of a series implies summability $[C ; k+\delta, q]$ of the series to the same sum for any $\delta>0$ and $q \leqslant p$. He has also noted that, for $k \geqslant 0$, summability $|C, k|$ of a series implies its summability $[C ; k, 1]$ to the same sum.

It will be shown that, with the above natural definition for summability $[C ; 0, p]$, certain known results involving multiplication of $[C ; k, 1]$-summable series with $k>0$ cannot be extended to include $k=0$.

2. The following results were proved by Winn $[2] ;$ his $[C, k]$ is the $[C ; k, p]$ as defined above with $p=1$.

Theorem 2. If $\Sigma u_{n}$ is summable $(C, k-1)$, where $k>0$, then it is summable $[C, k]$ to the same sum.

This result also holds for $k=0$. 
THEовем 5. If $\Sigma u_{n}$ is summable $[C, k]$ to $s$, and if $\Sigma v_{n}$ is summable $(C, l)$ to $t$, where $k>0$ and $l \geqslant 0$, then $\Sigma w_{n} \equiv \Sigma\left(u_{0} v_{n}+u_{1} v_{n-1}+\ldots+u_{n} v_{0}\right)$ is summable $(C, k+l)$ to st.

Theorem 6. $\Sigma u_{n}$ is summable $[C, k]$ to $s$ and $\Sigma v_{n}$ is summable $[C, l]$ to $t$, where $k>0$ and $l>0$, then $\Sigma w_{n}$ is summable $[C, k+l]$ to st.

3. The Case $k=l=0$ of Theorem 6 . If $\Sigma u_{n}$ and $\sum v_{n}$ are summable $[C, 0]$ then, by Hyslop's inclusion theorem, each of these series is summable $\left[C, \frac{1}{2} \delta\right]$ for any $\delta>0$ and so, by Winn's Theorem $6, \Sigma w_{n}$ is summable $[C, \delta]$. That $\Sigma w_{n}$ need not be summable $[C, 0]$ is shown by the following

Counterexample 1. Let

$$
u_{0}=u_{1}=0, \quad u_{n}=\frac{(-1)^{n}}{n \log n}(n \geqslant 2), \quad v_{0}=v_{1}=v_{2}=0, \quad v_{n}=\frac{(-1)^{n}}{n \log \log n} \quad(n \geqslant 3) .
$$

Then $\Sigma u_{n}$ and $\Sigma v_{n}$ are each summable $(C,-1)$, and so also summable $[C, 0]$, but

$$
\begin{aligned}
& \sum_{n=0}^{N} n\left|w_{n}\right|=\sum_{n=5}^{N} n\left|\sum_{r=2}^{n-3} \bar{r}-\frac{(-1)^{n}}{\log r)(n-r) \log \log (n-r)}\right| \\
& =\sum_{r=2}^{N}-3 \frac{1}{r \log r} \sum_{n=r+3}^{N} \frac{n}{n-r} \frac{1}{\log \log (n-r)} \\
& >\sum_{r=2}^{N-3} \frac{1}{r \log r} \frac{N}{N-r} \sum_{n=r+3}^{N} \frac{1}{\log \log (n-r)} \\
& >c N \sum_{r=2}^{N-3} \frac{1}{r \log r \log \log (N-r)}, \quad \text { where } c>0 \text {, } \\
& >\frac{c N}{\log \log N} \sum_{r=2}^{N-3} \frac{1}{r \log r} \\
& \sim c N \quad \text { as } N \rightarrow \infty
\end{aligned}
$$

Hence $\Sigma w_{n}$ is not summable $[C, 0]$.

It can, however, be proved that if $\Sigma u_{n}$ and $\Sigma v_{n}$ are summable $\left[(, 0]\right.$ then $\sum w_{n}$ is summable $(C, 0)$. For,

$$
\begin{aligned}
\sum_{n=0}^{N} n w_{n} & =\sum_{n=0}^{N} n \sum_{r=0}^{n} u_{r} v_{n-r} \\
& =\sum_{r=0}^{N} u_{r} \sum_{n=r}^{N}(n-r+r) v_{n-r} \\
& =\sum_{r=0}^{N} u_{r}\left\{r V_{N-r}+\sum_{s=0}^{N-r} s v_{s}\right\} \\
& =\sum_{r=0}^{N} r u_{r} V_{N-r}+\sum_{s=0}^{N} s v_{s} U_{N-s} \\
& =O\left\{\sum_{r=0}^{N} r\left|u_{r}\right|+\sum_{s=0}^{N} s\left|v_{s}\right|\right\} \\
& =o(N) .
\end{aligned}
$$


Since $\sum w_{n}$ is summable $[C, \delta]$ for any $\delta>0$, it is summable $(C, \delta)$ and hence summable $(C, 1)$. From the identity

$$
(N+1) W_{N}^{(0)}=W_{N}^{(1)}+\sum_{n=0}^{N} n w_{n}
$$

we have that $W_{n}^{(0)}$ tends to the same limit as $W_{n}^{(1)} / E_{n}^{(1)}$ as $N \rightarrow \infty$. Hence $\Sigma w_{n}$ converges.

4. Counterexample 2. Let $u_{0}=u_{1}=0, \quad u_{n}=(-1)^{n} /(n \log n) \quad(n \geqslant 2), \quad v_{0}=t$, $v_{1}=v_{2}=0, v_{3}=-3 / \log \log 3, v_{4}=4 / \log \log 4$,

$$
v_{n}=(-1)^{n}\left\{\frac{n}{\log \log n}-\frac{n-2}{\log \log (n-2)}\right\}, \quad \text { for } n \geqslant 5 \text {. }
$$

Then $V_{0}=V_{1}=V_{2}=t, V_{3}=t-(3 / \log \log 3)$,

$$
V_{n}=t+(-1)^{n}\left\{\frac{n}{\log \log n}-\frac{n-1}{\log \log (n-1)}\right\}, \text { for } n \geqslant 4 \text {. }
$$

Now $V_{n} \rightarrow t$ as $n \rightarrow \infty$, so that $\Sigma v_{n}$ is convergent, and hence summable [C, 1] to $t$. As before, $\Sigma u_{n}$ is summable $[C, 0]$; let its sum be $s$. Then

Hence, since

$$
W_{n}=\sum_{p=0}^{n} \sum_{q=0}^{n} u_{q} v_{p-a}=\sum_{r=0}^{n} u_{r} V_{n-r}
$$

$$
\begin{aligned}
\left(V_{r}-t\right) u_{n-r}=(-1)^{n}\left|V_{r}-t\right| \cdot\left|u_{n-r}\right|, & \\
\sum_{n=0}^{N}\left|W_{n}-s t\right| & =\sum_{n=0}^{N}\left|\sum_{r=0}^{n}\left(V_{n-r}-t\right) u_{r}+t\left\{\sum_{r=0}^{n} u_{r}-s\right\}\right| \\
& \geqslant \sum_{n=0}^{N}\left|\sum_{r=0}^{n}\left(V_{r}-t\right) u_{n-r}\right|-t \sum_{n=0}^{N}\left|\sum_{r=0}^{n} u_{r}-s\right| \\
& =\sum_{n=0}^{N} \sum_{r=0}^{n}\left|V_{r}-t\right|\left|u_{n-r}\right|+o(N) \\
& =\sum_{m=0}^{N}\left|u_{m}\right| \sum_{r=0}^{N}\left|V_{r}-t\right|+o(N) \\
& =\sum_{m=2}^{N-3} \frac{N-m}{m \log m \log \log (N-m)}+o(N), \quad \text { if } N \geqslant 5, \\
& =S_{1}+S_{2}-S_{3}+o(N),
\end{aligned}
$$

where

$$
\begin{aligned}
S_{1} & =N \sum_{m=2}^{N / 2} \frac{1}{m \log m \log \log (N-m)} \\
& \sim \frac{N}{\log \log N} \sum_{m=2}^{N / 2} \frac{1}{m \log m} \\
& \sim N \quad \text { as } N \rightarrow \infty \\
S_{2} & =N \sum_{m=\sum^{N}+1}^{N} \frac{1}{m \log m \log \log (N-m)}>0
\end{aligned}
$$

and

$$
S_{3}=\sum_{m=2}^{N} \frac{1}{\log m} \frac{1}{\log \log (N-m)}
$$




$$
=O\left\{\sum_{m=2}^{N-3} \frac{1}{\log m}\right\}=O(N) \text {. }
$$

Therefore $\sum_{n=0}^{N}\left|W_{n}-s t\right| \neq o(N)$, and $\Sigma w_{n}$ is not summable $[C, 1]$ to st. By Theorem 6, $\Sigma w_{n}$ is summable $[C, 1+\delta]$ to $s t$, so that, by the inclusion theorem, it cannot be summable [C, 1] to any sum other than st. Hence $\Sigma w_{n}$ is not summable $[C, 1]$ and so is not summable $(C, 0)$. This proves that Winn's Theorem 6 cannot be extended to the case $k=0, l=1$ and his Theorem 5 cannot be extended to include $k=l=0$.

Further, if $\Sigma v_{n}$ were summable $[C, 1-\delta]$ for some $\delta>0$, then, since $\Sigma u_{n}$ is summable $\left[C, \frac{1}{2} \delta\right], \Sigma w_{n}$ would be summable $\left[C, 1-\frac{1}{2} \delta\right]$ and hence summable $[C, 1]$. Since this is not the case, it follows that $\Sigma v_{n}$ is not summable $[C, 1-\delta]$, although it is summable $(C, 0)$ and $[C, 1]$. This shows that, when $k=1$, Winn's Theorem 2 is in a sense "best possible".

5. A multiplication theorem. Following the method of proof of Theorem 6 , we have the

Theorem. If $\Sigma u_{n}$ is summable $[C, k]$, where $k>0$, to $s$, and $\Sigma v_{n}$ is summable $|C, 0|$ to $t$, then $\Sigma w_{n}$ is summable $[C, k]$ to st.

LEMma (Winn [2]). If $\sum_{n=1}^{N}\left|\alpha_{n}\right|=o(N)$, then $\sum_{n=1}^{N} n^{p}\left|\alpha_{n}\right|=o\left(N^{p+1}\right)$ for $p>-1$.

Proof of the T'heorem. We have that $\Sigma v_{n}$ is summable $[C, 0]$. Suppose that $s=0$.

Equating coefficients of $x^{n}$ in the identity

$$
(1-x)^{-k} \sum_{n=0}^{\infty} u_{n} x^{n} \sum_{n=0}^{\infty} v_{n} x^{n}=\sum_{n=0}^{\infty} U_{n}^{(k-1)} x^{n} \sum_{n=0}^{\infty} v_{n} x^{n}
$$

we get

$$
W_{n}^{(k-1)}=\sum_{r=0}^{n} v_{r} U_{n-r}^{(k-1)}
$$

Then

$$
\begin{aligned}
w_{n}^{(k-1)} & =W_{n}^{(k-1)} / E_{n}^{(k-1)} \\
& =\frac{1}{E_{n}^{(k-1)}} \sum_{r=0}^{n} v_{r} E_{n-r}^{(k-1)} u_{n-r}^{(k-1)},
\end{aligned}
$$

and

$$
\begin{aligned}
\sum_{n=0}^{N}\left|w_{n}^{(k-1)}\right| & \leqslant \sum_{n=0}^{N} \sum_{r=0}^{n} \frac{\left|v_{r}\right| E_{n-r}^{(k-1)}\left|u_{n-r}^{(k-1)}\right|}{E_{n}^{(k-1)}} \\
& \leqslant \sum_{m=0}^{N} \sum_{r=0}^{N} \frac{E_{m}^{(k-1)}\left|r_{r}\right|\left|u_{m}^{(k-1)}\right|}{E_{m+r}^{(k-1)}} \\
& =\left|v_{0} u_{0}^{(k-1)}\right|+S_{\mathrm{L}}+S_{2}+S_{3},
\end{aligned}
$$

where

$$
\begin{aligned}
& S_{1}=\left|v_{0}\right| \sum_{m=1}^{N}\left|u_{m}^{(k-1)}\right|=o(N) \\
& S_{2}=\left|u_{0}^{(k-1)}\right| \sum_{r=1}^{N} \frac{\left|v_{r}\right|}{E_{r}^{(k-1)}}
\end{aligned}
$$


and

$$
=O\left\{\sum_{r=1}^{N} r^{1-k}\left|v_{r}\right|\right\}=o(N)
$$

$$
\begin{aligned}
S_{3} & =\sum_{m=1}^{N} \sum_{r=1}^{N} \frac{E_{m}^{(k-1)}\left|v_{r}\right|\left|u_{m}^{(k-1)}\right|}{E_{m+r}^{(k-1)}} \\
& <K \sum_{m=1}^{N} \sum_{r=1}^{N} \frac{m^{k-1}\left|v_{r}\right|\left|u_{m}^{(k-1)}\right|}{(m+r)^{k-1}}
\end{aligned}
$$

To show that $S_{3}=o(N)$ it suffices to prove the result for any particular $k_{0}>0$; it will then follow for all $k \geqslant k_{0}$, since $\{m /(m+r)\}^{k-1}$ is a decreasing function of $k$. If $k \leqslant 1$, we have, since $\Sigma v_{n}$ is $|C, 0|$,

$$
\begin{aligned}
S_{3} & >K(2 n)^{1-k} \sum_{m=1}^{N} m^{k-1}\left|u_{m}^{(k-1)}\right| \sum_{r=1}^{N}\left|v_{r}\right| \\
& =O\left\{N^{1-k} \sum_{m=1}^{N} m^{k-1}\left|u_{m}^{(k-1)}\right|\right\} \\
& =O\left(N^{1-k} N^{k}\right)=o(N),
\end{aligned}
$$

by the Lemma. $\Sigma w_{n}$ is then summable $[C, k]$ to 0 .

If $s \neq 0$, put $u_{0}^{\prime}=u_{0}-s, u_{n}^{\prime}=u_{n}(n>0)$, so that $\sum u_{n}^{\prime}$ is summable $[C, k]$ to 0 . Then $\Sigma w_{n}^{\prime}$ is summable $[C, k]$ to 0 . But $\Sigma w_{n}=\Sigma w_{n}^{\prime}+s \Sigma v_{n}$ and $\Sigma v_{n}$ is summable $[C, k]$ to $t$. . Hence $\sum w_{n}$ is summable $[C, k]$ to $s t$.

Whether this result remains true when $k=0$ is at present unsettled.

\section{REFERENCES}

1. J. M. Hyslop, Note on the strong summability of series, Proc. Glasgow Math. Assoc., 1 (1952), 16-20.

2. C. E. Winn, On strong summability for any positive order, Math. Zeit., 37 (1933), 481-492.

UNIVERSITY OF THE WITWATERSRAN D

JOHANNESBURG 